\title{
The Traditional Chinese Medicine of Anti-treatment and Anti-treatment Method
}

\author{
M.S. MIAO, S. TIAN, M. BAI, J.J. JIA, T. TING \\ Henan University of Traditional Chinese Medicine, Zhengzhou 450008, China
}

\begin{abstract}
Anti-treatment is an important treatment rule of traditional Chinese medicine treatment disease, but the anti-treatment method as unique treatment method of traditional Chinese medicine, it is one of the important methods of TCM clinical treatment of the disease, it has very different ideas with Western medicine in the treatment, it also can be used to complement Western medicine "treatment resistant inadequate". This paper by combing the literature, summarized the Anti-treatment method of origin, significance and changes, clarify the modern meaning and study of Chinese modern anti-treatment method, clarify the theoretical basis and mechanism of anti-Chinese medicine treatment disease method, promote the development of the Antitreatment method of traditional Chinese medicine, and promote the development of Chinese medicine treatment of modern anti-conquer disease.
\end{abstract}

KEYWORD: Chinese Anti-treatment; meaning; the efficacy of Chinese medicine; modern research

\section{INTRODUCTION}

Anti-treatment is a method commonly used in ancient Chinese Medicine. It is the traditional Chinese medicine dialectical way of thinking and a kind of special therapeutic principle in traditional Chinese medicine. The first is contained in the "PlainQuestions", the emperor said: "What is indicative treatment and contrary treatment ". Qi Bo said:" Indicative treatment is the treatment, contrary treatment is the treatment contrary to the routine, obey more or less, according to the actual situation[1]. The doctor of traditional Chinese medicine clinical dialectical treatment has a complicated thing. We must seize the essential characteristics of the disease, the correct treatment Reverse treatment is a special method of Chinese medicine treatment of the disease, for the condition is complex, serious, etiology and pathogenesis and the external symptoms are not consistent with the disease. This paper discusses the significance of the anti-Chinese medicine therapies, and changes in governance and modern research of traditional Chinese Medicine Anti treatment.

\section{THE TREATMENT OF TRADITIONAL CHINESE MEDICINE}

\subsection{The meaning of Chinese medicine treatment and reverse treatment}

The development of therapeutics of traditional Chinese medicine, has a long history and goes back to ancient times. In the long process of development, therapeutics of traditional Chinese medicine constantly enrich and also has its own unique characteristics. The cure of traditional Chinese medicine of traditional Chinese medicine (TCM) is one of the important therapeutic principle of treating diseases, past dynasties doctor understanding of its concept and essence there are a lot of controversy anti-treatment is the external manifestation of obedience to the rule of illusion disease a treatment rule, the nature of the drug and the disease is often reflected pseudo symptoms consistent, so it is also called from the cure. From the nature of disease, refers to obey by prescription of illusion, consistent with the illusion of the disease, its essence, or in the cure for this rule under the guidance of the disease, according to the nature and the method of treatment, it is the result of "searching for root cause of disease".

In the progress of the disease process, when in the process of complex pathological, abnormal development of the disease, illness is complex, some diseases of clinical manifestation and its essence is 
inconsistent, there will often be some different from the general law of development of the disease as a special permit, the illusion occurs, such as "excess syndrome with pseudo-deficiency symptoms, pseudo-excess symptoms appearing in extreme deficiency syndrome", etc. If this time also adopts a routine treatment. Such as "purge the excess", "then fill the empty" principle will be worse, even endanger the lives. At this time should be used in anti- treatment method. On the surface treatment prescription and disease from the phenomenon of phase, and the essence and disease phase inverse, namely anti-treatment, this is the meaning of the treatment in the therapeutic principle treatment. In fact, there is also under the direction of law searching for root cause of disease. A method treatment is carried out, for the nature of disease. It is still "searching for root cause of disease" in essence. There are some commonly anti-treatment methods:"treating pseudo-heat syndrome with hot therapy" "treating pseudo-cold with cold drugs" "treating obstruction with tonics" "treating diarrhea with purgative."

Treating pseudo-heat syndrome with hot therapy referred to the heat treating heat syndrome treatment, which uses warming prescription treat presenting with symptoms of false heat drug treatment. It is suitable for the yin-cold excess, Yang at outside of pseudo pyretic cold syndrome. If using Decoction of Ephedra, Aconite and Asarum soup treatment of fever, sore throat, effect is good[2]. And such as the use of "treating pseudo-heat syndrome with hot therapy" with the rule of dredging collaterals with pungent-warm herbs treat scleritis, malnutrition with Decoction of Ephedra, Aconite and Asarum soup for scleritis, curative effect is distinct [3]. Shanghai Shuguang Hospital famous old Chinese doctor Mr. Liu Heyi once while using the "treating pseudo-heat syndrome with hot therapy" anti-treatment method with Ephedra Decoction with Bighead Atractylodes treated with lobar pneumonia pungent-warm herbs agents to treat, was along its potential[4]. "Treating pseudo-cold with cold drugs" means to cure cold, with cold medicine cure the false and cold symptoms, apply to the hot extremely exuberant, the really hot fake and cold syndrome of yang gas too strong to block out the yin gas. Such as "ShangHanLun" says: the syncope of treatise and pulse slippery, there are hot, the white tiger soup can cure. "Treating obstruction with tonics" refers to fill open plug, which uses drugs to treat tonic syndromes have symptoms blocking barrier for virtual and closed due to the resistance of pseudo-sthenia deficiency, such as the management in the soup cure spleen Fu Man of the symptom Chinese medicine practitioners such as Wang Shaohua used Buzhongyiqi decoction to cure women with postpartum blood deficiency constipation, Buzhongyiqitang Qi intestines, lung and spleen so angry with the charge, you pass a strong, stool, socalled treating obstruction with tonics"". Treating diarrhea with purgative is due to pass through the pass, that the use of medication can pass through solid profits have real reapseudo sthenia deficiency, with a profit of delirium Dachengqi decoction rule under license. As Xusheng Sheng Guadi and red bean with each $2 \mathrm{~g}$, finely and evenly, decoction delivery service, treatment overeating, excessive drinking may cause vomiting, so make the best Sushi toxins, induce vomiting by evil, so evil is from the best and vomiting .

\subsection{The development history of the method of traditional Chinese medicine of Anti-treatment}

In ancient times, witch and medical were homologous, witch doctor was the earliest in the sense of the doctor, "Origin of Chinese Characters" said The doctor was the treatment of workers, in the ancient witch doctor first work as ancient doctors, in the "Guang Ya, interpretation of the words": "The doctor was Wizard". Therefore, in the ancient times medicine and witch was communicated. About in The Ritual of Zhou, medicine gradually separated from the cult, in the "rites", had taken the wizard and physician listed separately in different official system. To the Spring and Autumn Spring and Warring States Period. Ancient medical BianQue puts forward who believed witch doctors do not believe the medical did not be cured. This was the first in a declaration on the separation of medicine and medical witch. To the warring states period, the birth of Huangdi Neijing, both the disintegration of the witch and doctor. The Chinese medicine gradually formed. And the treatment of traditional Chinese medicine as an important therapeutic principle of TCM, in the early stages of the development of Chinese medicine, that generations of physicians had realized the function of the treatment in the treatment of traditional Chinese medicine.

The spring and autumn period and the warring states period, the warring states period, the advent of Huangdi Neijing, which initially formed the therapeutic principle of dialectical treatment, provided theoretical guidance for the development of therapeutics of traditional Chinese medicine and the basis, and in NeiJing says"mild disease being routinely treated, serious cased with pseudosymptoms (cold or heat)treated with herbs, the nature of which is consistent to pseudo-symptoms inverse normal treatment, inverse normal treatment, the followers to, it first put forward the antitreatment word.

After the unification of Qin and Han Dynasty, the medical system was further mature, the emergence of a large number of medical prescription and the medical classics, preliminary established the whole 
dialectical theory of governance principles, Zhang zhongjing's Treatise on Cold-Induced and Miscellaneous Diseases, on the basis of inheriting the idea of NeiJing pioneered the TCM therapeutic principle of combining theory and practice of the foundation. "Treatise on Cold-Induced and Miscellaneous Diseases" to use anti cure very flexible, In the "Treatise on febrile diseases, differentiation of the sun pulse and symptoms and cure": "the patient to get hot, anti cloth, heat in the skin, cold in the bone marrow; body chill, anti desire to wear clothes, cold in the skin, marrow heat also". When the disease occurrence contradiction, easy to fake easily confused, it must be explored the essence through the phenomenon, disease, treatment was apparent signs of disease and cure for obedience, called anti-treatment method, was a concrete manifestation of anti-treatment.

During the Sui and Tang Dynasties, with the development of economic prosperity, Chinese medicine was also continuous improvement and development, governance theory also constantly enriched, the Tang Dynasty Wang Bing further developing the "classics" of the Yellow Emperor is thought, in the "prime department to really want to big theory" in the note said "of the cold and heat of Yin, however, and cold person taking Yang", put forward "strong Lord of the water, for the sun; fire and the source of benefits, in order to eliminate the cloud" theory, he defined as "treatment of anti disease and anti treatment of people from gas".

During the Song Dynasty, Chinese medicine theory had been constantly deepened and refined, for the discrimination of true and false cold and heat were further enriched. Such as Xu Shuwei of the Song Dynasty analyzed true and false cold and heat, further enriched the theory of normal treatment and anti-treatment. During the period of Jin Yuan, one of the "Four of Jin Yuan" Li Gao, used " warm tonic " method to further promoted the application of treating obstruction-syndrome with tonics, enriched and perfected the theory of anti-treatment, and the Buzhong Yiqi Tang created by him, had been applied and circulated so far.

During the Ming Dynasty, there was more accurate documentary records of normal treatment and anti-treatment expression. Zhang Jingyue's "Jingyue encyclopedia, volume one, the first part of Chuan Zhong Lu, on the treatment of article ten" said: "the treatment had anti and normal methods, so the cold and heat were the true or the false. This was the keynote of NeiJing. If treated heat in cold or treated cold in heat, this was called normal treatment, so the normal was the anti. Heated in heat or cold in cold, this was called anti-treatment, so the anti was the normal". Another saying: Treating diarrhea with purgatives, such as savings hot inside, great cold stagnation inside, stagnation and retention, diarrhea more than. The people of cold stagnation were taken heat to removal, the people of the thermal hysteresis were taken cold to remove. This was method of treating diarrhea with purgatives. In addition, Ma Shi noted: "what need to V main body, the heat treatment, such as cold, cold and heat treatment to that. Medicine should be what use, will be at the head, as a result of the cold because of the heat, Yinsaiyintong called", thought that the Chinese medicinal anti-treatment was using corrigent.

During the Qing Dynasty, Chinese medicine was further deepen, research on reverse treatment of traditional Chinese medicine was more and more clear. According to Wu Jutong said, " because of the much hot Yangming, Body fluid boring, water was not enough to ship, the people whose the accumulation of feces could not be discharged should increase liquid ", cast a sweet and cold , salty and cold moistening agent. Such as fluid increasing Decoction, supplied intestinal fluid, moisten intestines, to achieved "increasing water to boat ", "to make up for the purpose of glycerine ". That was so-called "treating obstruction-syndrome with tonics".

The period of the Republic of China, with the introduction of Western medicine, Chinese medicine in the clinical application of reverse treatment were also increasingly expanding. Zhang Xichun who writed the book of "Traditional Chinese and Modern Western Medicines" created a plaster aspirin soup, beginning with aspirin in the treatment of the disease, the effect was very good, but did not have the effect of cleaning inside of the body, if sweating, immediately with one Liang gypsum decoction, took a hot drink, sweated more, as could clear heat, so the disease could be cured. Or taking aspirin in gypsum decoction could make the disease be cured. In clinical practice as a basis, created the paradigm of both using chinese and western medicine, and also expanded the application of anti-treatment of traditional Chinese Medicine.

\section{CHINESE ANTI-TREATMENT METHOD OF MODERN MEANING}

With the development of modern medicine, there are different views on the understanding of traditional Chinese medicine of anti-treatment, such as somebody said the anti cure "from false, namely rule the disease from feint", but others said "essence of anti-treatment is normal treatment", etc. Each expresses his own views. On the anti-treatment opinions can be divided into three kinds: The first kind is that anti-treatment is substantially normal treatment, there is no so-called anti-treatment, Such as the study of " Chinese medicine research" written by Jiang Chunhua in 1983 said: These are the dialectical problems, seek the truth from the Illusion is still normal treatment, no treatment of the so- 
called anti. The second is that anti-treatment methods are substantially same with normal treatment, but there are still some differences. Such as the book of "Chinese medicine therapy" written by Sun Guojie in 1990 said: Anti-treatment should belong to the normal treatment in the strict sense, but in some special cases is a therapy of curing along its symptoms and signs. Third is that normal treatment and anti-treatment are both cure for the essence, there is a difference between the two. Such as the "basic theory of traditional Chinese medicine" written by Wu Dunxu in 1995 said: both are the cure for this category, but there are some differences between revolt and obedience in the nature of drugs, utility medication and disease. In addition, Their suitable diseases and syndromes are different. if the nature of the disease and the clinical manifestation is not completely consistent, using anti-treatment method. Anti-treatment methods select the drugs whose nature is similar to the disease, development of obedience, create the internal environment which can fully display symptoms, strengthen the local feedback information, speed feedback speed, excitation physiology "adversarial system", to create conditions for the establishment of the new steadystate physiological system. In addition, using corrigent and anti-treatment of the two concepts are different, anti-treatment is the treatment principle [5], and using corrigent is a medication way of reverse treatment, can also be understood as extension of reverse treatment. That is, under the guidance of the principles of treatment, implied Herbal Prescription and fried with taking methods. Using corrigent drugs have two kinds of cases in clinical application, compatibility of using corrigent and compatibility of taking method, such as when supply Yin, proper compatibility Bhd drug, make Yin's source constantly with the help of Yang; When supply Yang, in appropriate compatibility yin-tonifying drug, make Yang got endless growth differentiation with the help of Yin.

\section{CHINESE ANTI-TREATMENT METHOD OF MODERN RESEARCH}

With the progress of science and technology, the modern research of traditional Chinese Medicine Anti treatment also increasingly increased, traditional Chinese medicine therapy for the treatment of anti chronic kidney disease[6], prostatitis, prostatic hyperplasia, diarrhea, especially the application in the treatment of women's diseases, such as perimenopausal syndrome, irregular menstruation, breast pain, bleeding, habitual abortion. Such as red Siwu Decoction Combined with Shixiaosan plus Salvia miltiorrhiza, motherwort treating metrorrhagia and metrostaxis with $\mathrm{Xi}$ Liang Cheng old doctor of traditional Chinese medicine, medication 6 agents, blood leakage is stopped. Zhao Shengquan took Bazhen soup tonifying spleen for nourishing gas in treating long cough, immediately successful. Zhu Fengming to benefit the treatment of Otolaryngology closed resistance caused due to false true false positive and effective. Below traditional Chinese Medicine Anti-treatment of perimenopausal syndrome with modern research described.

Perimenopausal syndrome of multiple is between $45 \sim 55$ years old, the doctor of traditional Chinese medicine thinks kidney essence is the life activities of the human body. Perimenopausal women, the deficiency of kidney qi, Ren Chong channel, menstrual disorder appears, and menopause, accompanying also appear a series of insufficiency of kidney water, fire endogenous and other syndromesbecause of deficiency of kidney essence, such as hot flushes, sweating, five upset hot, Yaoxisuanruan, insomnia and dreaminess etc. Antitreatment method complied the external appearance of diseases, such as sweating, hot flashes, to treat the disease by using Bhd drug temperature of Cynomorium songaricum Rupr, Longspur epimedium, namely with the heat of drugs to treat with false heat symptoms of the disease, has obtained the good curative effect. According to the clinical perimenopausal performance, it was divided into the liver and kidney yin deficiency, kidney yin and yang two deficiency, heart kidney incoordination, spleen kidney yang deficiency and liver gas stagnation[7]. Proved by clinical and experimental proof, kidney yang drugs with in vivo quasi estrogen like effects[8], traditional Chinese medicine in the treatment of perimenopausal syndrome of traditional Chinese medicine is mainly of kidney yang, such as velvet, Cynomorium, Fructus Psoraleae, barrenwort, morinda. The ethanol extract of Morinda officinalis days with similar estrogen like effects, can improve the level of estrogen in vivo, reduce the degree of atrophy of uterus[9]; Experimental study on otherwise found, 18 month old female rats' serum E2 levels are elevated in given Erxian Decoction, decrease the content of FSH and $\mathrm{LH}$, that the treatment of reproductive endocrine mechanism of perimenopausal syndrome. The study found that epimedium total flavonoids can significantly increase the apparent surface density and bone density of femur of rats, but not increase uterine coefficient and serum estradiol level, and have the trend of improving the bone $\mathrm{Ca}$ and bone $\mathrm{P}$. Studies have shown that guilubushen pills could promote the growth and development of reproductive system in ovariectomized mice[10].

\section{SUBMISSION OF MATERIAL}

As an old subject, Traditional Chinese medicine offers guidance on the application of traditional 
Chinese medicine. For example, as early as in the "Huangdi Neijing" on the proposed requirements for medical personnel, the requirements of medicine show that the medical personnel should understand the patient's natural environment, but also understand the patient's social interpersonal things, like the social politics, economy, culture folk customs, etc. , even the personal political status, family encounter and personal experience, because of all these factors and mental and physical health are closely related. Treatment should not only adapt to the laws of nature or suit one's measures to local conditions, but also pay attention to adjust the patients' mental emotional and physiological function because of abnormal social factors, flexibility in the use of Chinese medicine treatment principle.

TCM holds that the human body is in a state of unity of opposites mutual connection, mutual constraints named "Balance of yin and Yang, Spirit will be cured", is the human body health state. When the body imbalance of yin and Yang, will break the balance state of body balance of yin and Yang, and thus caused by pathological changes in the body. Such as women in the normal condition, secretion of estrogen and androgen in vivo is proportional to. Once the balance is broken, the body's estrogen and androgen secretion is imbalanced, which cause a series of pathological state. Such as adolescence, teenagers growing nevus on faces is because hormone secretion in vivo is not stable, normal estrogen and high androgen. Perimenopausal syndrome is due to the body of excessive low secretion of estrogen, out of balance of yin and Yang, and neurotransmitters, hormones, immune mediators cause reduced symptoms.

At present, because of the people fanaticism pursued the traditional Chinese medicine, the application of traditional Chinese medicine therapy in the anti-treatment of some difficult miscellaneous diseases, has obtained the good treatment effect, make the study of reverse treatment is also increasingly increased. But in the actual application of reverse treatment also exist some problems, such as the anti drug and disease treatment with multiple similar, easy to appear the body reaction and side effect, it also has some limitations in clinical application. Therefore, in the application of reverse treatment, should be combined with other treatment such as rousing, regulating yin and Yang, suiting measures to the timing, the people and local condition, accurately grasp the opportunity to drugs, drug dosage, drug treatment of the severity and length of time to wait, should according to the severity of the disease and the strength of the gas, the specific analysis, flexible to grasp, to reach the treatment of disease, improve the curative effect. In addition, through the multi-disciplinary, multi angle analysis of anti-treatment method, grasp the essence, explore the treasure house of the motherland medicine, inheritance of the medical thought, formed the theoretical system characteristic, promote the diversification of Chinese medicine treatment methods, dosage form of individual, the maximum effect, promote the use of Chinese medicine treatment of the anti-treatment methods in the modern disease treatment, development prospect better.

\section{REFERENCES}

[1] Fan, P. Hou, M. Yang, CY. et al. 2013. Application of Gang Shunkui director of clinical experiences of reverse treatment. Yunnan Journal of traditional Chinese medicine34 (5): 3-5.

[2] Yu, LH. 2013. Classical Prescriptions "treat heat with heat" treatment of fever case two. Journal of Chengdu University of Traditional Chinese Medicine36(4):110-111.

[3] Xie, YI. Wan, WR. 2011. Discussion of cure rickets with Mahuang Fuzi Xixin Decoction from the method of "treat heat with heat". Herald of Medicine-classic research10 (2):11-12.

[4] Lian, JC. 2004. The homeopathic medicine and Chinese medicine treatment of anti. Jiangxi Journal of traditional Chinese medicine35 (6): 21-22.

[5] Guo, XJ. Zhang, YW. Zhao, JW. 2011. Zhang Jingyue's anti Zuo thought. Shaanxi traditional Chinese medicine32 (1): $127-128$.

[6] Zhao, J. 2012. Sun Wei use anti treatment of chronic kidney disease proved cases. Jiangsu Journal of traditional Chinese medicine44 (1): 43-44.

[7] Chen, WX. Huang, GP. 2013. Progress in the treatment of climacteric syndrome of traditional Chinese medicine. Journal of clinical rational drug use6 (2): 176-177.

[8] Wei, ZZ. Miao, MS. 2013. The analysis synthesis of Nourishing Kidney Yang treatment of perimenopausal syndrome characteristics. Journal of Traditional Chinese Medicine(11): 1688-1691.

[9] Wang, Y. Zhang, Q. 2011. Experimental study on the estrogen like effect of Morinda officinalis. Lishizhen medical drug22 (3):527-528.

[10] Yi, LN. Hu, YJ. Wang, JH. 2012. Overview of party research Yang tonifying kidney. lishizhen medicine and Materia Medica Research 23 (3):728. 